\title{
External Environment Dependent Spin and Orbital Exchange Interactions
}

Eunsung Jekal ${ }^{*}$

THE Institute, Department of Mathematics, Daseon Education, Ulsan, South Korea

* Corresponding author email: everjekal@gmail.com

Received: 27 May 2020 / Revised: 19 June 2020 / Accepted: 18 July 2020 / Published: 29 July 2020

\section{ABSTRACT}

We present a set of equations expressing the parameters of the magnetic interactions of an electronic system. This allows to establish a mapping between the initial electronic system and a spin model including up to quadratic interactions between the effective spins, with a general interaction (exchange) tensor that accounts for anisotropic exchange, Dzyaloshinskii-Moriya interaction and other symmetric terms such as dipole-dipole interaction. We present the formulas in a format that can be used for computations via Dynamical Mean Field Theory algorithms.

Keywords: Spin, Orbital, Dzyaloshinskii-Moriya interaction

\section{Introduction}

Describing a solid in terms of its magnetic properties requires the knowledge of an effective spin model which displays the same interesting physical properties as the many-electron Hamiltonian whose exact solution would give the complete description of the system. The determination of the form of the effective spin model and of the strength of the interactions between the constituent spins starting from the initial electronic model is, in general, a complicated many-body problem [1-5]. We have recently derived expressions for the parameters of the magnetic interactions within an extended (multi-orbital) Hubbard model [6], in the presence of arbitrary relativistic couplings affecting the electronic degrees of freedom (such a spin orbit, magnetic anisotropy, Zeeman coupling with an external magnetic field). The formulas presented in Ref. [6], after neglecting the vertices of two-electron Green's functions, are expressed in terms of single-electron (but fully interacting) Green's functions $G$ and the single-electron (hopping) Hamiltonian $T$. The use of presentation via $T[6,7]$ for computations related to real materials requires the additional step of a tight-binding parametrization, which is implemented only in some methods of electronic structure calculations. On the other hand, a presentation of the formulas in terms of Green's functions $G$ and self-energies $\Sigma$ would make them more suitable for implementation via Dynamical Mean Field Theory (DMFT), since any DMFT calculation deals with $G$ and $\Sigma$. Writing the parameters in a way that explicitly exhibits self-energies, analogous to what was done in Refs. [1,2], also allows to explicitly include the approximation of local self-energy, which is the key assumption of DMFT $[8,9,10,11]$.

Spin-orbit interaction in two-dimensional materials brings many exotic phenomena to be explored. In transition-metal dichalcogenides, large spin-orbit interaction induced band splitting in both conduction and valence band gives rise to valley Hall effect $[17,18]$ and unconventional quantum Hall effect $[19,20]$. Recently, band inversion caused by spin-orbit coupling proximity effect 5 is observed in graphene/ $\mathrm{WSe}_{2}$ heterostructure [21]. 
Spin-orbit interaction has been extensively studied in III-V semiconductors like InGaAs/InAlAs quantum wells for spintronic applications [22,23]. Chiral crystals with spin-orbit interaction are predicted to host Kramers-Weyl fermions and other topological quantum properties [24].

We here present the adaptation of the formulas for the exchange tensor to this scheme.

\section{Method and discussion}

We consider the extended multi-orbital Hubbard Hamiltonian,

$$
\mathrm{H}=\sum_{\mathrm{o}, \sigma, \mathrm{m}} \sum_{\mathrm{o}^{\prime}, \sigma^{\prime}, \mathrm{m}}{ }^{\prime} \varphi_{\mathrm{o}, \sigma, \mathrm{m}}^{\dagger} \mathrm{T}_{\mathrm{o} \prime, \sigma^{\prime}, \mathrm{m} \mathrm{m}^{\prime}}^{\mathrm{o}, \mathrm{m}} \varphi^{\mathrm{o}, \sigma^{\prime}, \mathrm{m} \prime}+\mathrm{H}_{V},(1)
$$

where the field operator $\varphi_{\mathrm{o}, \sigma, \mathrm{m}}^{\dagger}$ creates an electron with quantum numbers $o, \sigma, m$ refers to a set of the orbital indices (for a basis of localized Wannier wave functions, these are the atom index $a$, the principal atomic quantum number $n$ and the angular momentum quantum number l:o $\equiv a, n, l$, while $\sigma \uparrow, \downarrow$ and $m \in l$, $l+1, \ldots, l$ are the third components of the intrinsic spin and orbital angular momenta, respectively. Local angular momenta are measured with respect to local reference frames, which depend on $o$ and might not be collinear [12]. The single particle Hamiltonian matrix $\mathrm{T}_{\mathrm{o}^{\prime}, \sigma, \sigma^{\prime}, \mathrm{m}}^{\mathrm{O}, \sigma, \mathrm{m}}$ is completely arbitrary, so it can include any relativistic single-electron terms (Zeeman coupling, spin-orbit, magnetic anisotropies). The interaction Hamiltonian $\mathrm{H}_{V}$ is assumed to be rotationally invariant.

The goal in Ref. [10] was to map the model given by Eq. (1) onto an effective model of classical spins $e_{o}$ including up to (arbitrary) quadratic interactions, with Hamiltonian,

$$
H_{\text {spin }}=\sum_{o} e o \cdot B o+\frac{1}{2} \sum_{o, o l} \sum_{a, a \prime \prime} e_{o, a} e_{o \prime, a \prime} H_{o o^{\prime}}^{a a \prime^{\prime}}
$$

determined by the exchange tensor $H_{o o^{\prime}}^{a a \prime}=H_{o \prime o}^{a \prime a}$ (here and in the following $a$ and $a^{\prime}$ are used to denote the space coordinates, e.g.x,y,z) and the effective magnetic field Bo. It is convenient to decompose the exchange tensor into the three vectors $J_{o o^{\prime}}=J_{o \prime o}$ (anisotropic exchange), $D_{o o^{\prime}}=-D_{o \prime o}$ (DzyaloshinskiiMoriya interaction), and $C_{o o \prime}=C_{o \prime o}$ (symmetric non-diagonal exchange), defined as

$$
\begin{gathered}
J_{o o \prime}^{\alpha} \equiv H_{o o \prime}^{\alpha \alpha},(3) \\
D_{o o^{\prime}}^{\alpha} \equiv \frac{1}{2} \sum_{\alpha \prime \alpha \prime \prime} \epsilon^{\alpha \prime \alpha \prime \prime \alpha \prime \prime \prime} H_{o o^{\prime}}^{\alpha \prime \alpha \prime \prime}, \\
C_{o o \prime}^{\alpha} \equiv \frac{1}{2} \sum_{\alpha \prime \alpha \prime \prime}\left|\epsilon^{\alpha \prime \alpha \prime \prime \alpha \prime \prime \prime}\right| H_{o \prime^{\prime}}^{\alpha \prime \alpha \prime \prime},
\end{gathered}
$$

where $\epsilon^{\alpha \prime \alpha \prime \prime \alpha \prime \prime \prime}$ is the completely anti-symmetric tensor of rank 3 . The Heisenberg model is obtained as the particular case in which $H_{o o^{\prime}}^{\alpha \alpha \prime} \equiv \delta^{\alpha \alpha^{\prime}} J_{o o}$.

To perform the mapping, in Ref. [5,9], we have derived the response of the thermodynamic potential of the electronic system under small spatially dependent rotations of the spin quantization axes associated with each orbital spinor denoted by $o$, up to second order in the rotation angles. The derivation of such response involves path integration over the fermionic fields after the introduction of auxiliary bosonic degrees of freedom which express the amplitudes of rotations from an initial spin configuration; the coefficients of the interactions between the remaining bosons are put in correspondence with the parameters of the spin model (2) by imposing that the thermodynamic potential of the spin system after the spin rotations is equal to that of the electrons. Excluding the vertex contributions, the parameters of the spin model are expressed in terms of single-electron Green's functions (which of course include interaction effects) and the single-particle part of the electronic Hamiltonian, $T$ [13-15].

This procedure is similar to the one previously adopted in Refs. [4,5] for the case of quenched orbital moments, but in Ref. [12], we have considered rotations of the local total spins $S_{o}^{2}=l_{o}^{2}+s_{o}^{2}$, where $l_{o}^{2}$ and $s_{o}^{2}$ are orbital and spin, respectively, the orbital and intrinsic angular momenta associated with the states 0 . More precisely, we have considered rotations in the space of the single-particle eigenfunctions of $S_{o}^{2}$ and 
Eunsung Jekal, J. Mod. Sim. Mater.; Vol. 3 Issue 1, pp: 79-83, 2020

$S_{o}^{Z}$, analogous to Ref. [9], while in Refs. [4,5] the rotations affected the space of eigenfunctions of of $s_{o}^{2}$ and $s_{o}^{Z}$. This allowed us to obtain formulas for the exchange tensor that can be separated into contributions coming from the interactions between spin-spin, orbital-orbital, or spin-orbital degrees of freedom of the electrons; for example, for the anisotropic exchange parameters we have

$$
J_{o o^{\prime}} \equiv J_{o o^{\prime}}^{\text {spin-spin }}+J_{o o^{\prime}}^{\text {orbit-orbit }}+J_{o o^{\prime}}^{\text {spin-orbit }} \text {, }
$$

The terms labelled as spin-spin or spin are those contributions to magnetic interactions that would arise if we kept the orbital magnetic moments quenched, i.e., if we rotated only the intrinsic spin- spinors. Analogously, the terms labelled as orbit or orbit-orbit arise if we rotate only the orbital magnetic moments, keeping the intrinsic spins quenched. The terms labelled as spin-orbit arise only when the total local magnetic moments are rotated; these terms should not be confused with spin-orbit coupling, which contributes in general to all terms [1-10].

It should be noted that the possibility of rotating the total local spins is not applicable within Density Functional Theory (DFT) formulations, where observables are expressed in terms of the charge density and the intrinsic-spin density. The possibility of rotating local total spin is related to the representation of the electronic Hamiltonian in terms of localized wave functions, which implies a higher number of degrees of freedom with respect to DFT (related to the fact that the set of localized states would be over complete in theory, or not even complete in practice due to truncation).

The computation of the magnetic parameters via DMFT is greatly simplified if they are formulated in terms of single-particle Green's functions and self-energies $\Sigma$ in magnetically ordered states, since this avoids the initial step of a tight-binding parameterization of the single-electron Hamiltonian, T. To remove $T$ and introduce $\Sigma$, we use the equations of motion for Matsubara Green's functions (Dyson equations), which we write in general matrix notation as

$$
\begin{gathered}
(\omega-i \mu) G(i \omega)+i T \cdot G(i \omega)=1-\sum(i \omega) \cdot G(i \omega), \\
(\omega-i \mu) G(i \omega)+i G(i \omega) \cdot T=1-G(i \omega) \cdot \Sigma(i \omega) \cdot(7)
\end{gathered}
$$

These equations hold for the Matsubara Green's functions defined according to the following convention: $G_{2}^{1}(\tau) \equiv-\mathrm{i}<\mathrm{T} \psi^{1}(\tau) \Psi_{2}^{\dagger}>\equiv \frac{1}{\beta} \sum \omega G_{2}^{1}(\mathrm{i} \omega) \mathrm{e}^{\mathrm{i} \omega \tau}(8)$

Where $\omega=(2 \mathrm{n}+1) \pi / \beta$ is a fermionic Matsubara frequency, $\beta$ being the inverse temperature. As a particular case, the single-electron density matrix is given by

$$
\varrho \equiv-i G\left(\tau=0^{-}\right)=-\mathrm{i} \frac{1}{\beta} \sum \omega \mathrm{e}^{\mathrm{i} \omega+} G(\mathrm{i} \omega) \cdot(9)
$$

We now have to distinguish between the magnetic parameters that can be computed from the second order response in the rotation angles and those which are computed from the first-order response. From Ref. [12], we note that the former terms can all be written in terms of the following quantity: $\mathrm{F}_{\mathrm{O} \alpha, \mathrm{o} / \alpha}^{\mathrm{XY}}$ where $\mathrm{X}, \mathrm{Y} \in[$ spin,orbit] refer to either spin- or orbital-related terms, that is,

$$
\mathrm{S}_{\mathrm{o} \alpha}^{\mathrm{spin}} \equiv \mathrm{S}_{\mathrm{o} \alpha} \equiv \frac{1}{2} \sigma_{\mathrm{o} \alpha}, \mathrm{S}_{\mathrm{o} \alpha}^{\mathrm{orbital}} \equiv \frac{1}{2} l_{\mathrm{o} \alpha},(10)
$$

where $\mathrm{S}_{\mathrm{o} \alpha}$ is an intrinsic spin matrix (sigma $\mathrm{o} \alpha$ is a Paulimatrix), while $l_{\mathrm{o} \alpha}$ is an orbital angular momentum matrix. In Eq.(10), we have used the notation $\{A ; B\} \equiv A \cdot B+B \cdot A$ to denote the anticommutator of the matrices $\mathrm{A}$ and $\mathrm{B}$; in the following we will also make use of $[\mathrm{A} ; \mathrm{B}] \equiv \mathrm{A} \cdot \mathrm{B} \quad \mathrm{B} \cdot \mathrm{A}$ to denote the commutator. From the Dyson equations (5), we have (the frequency arguments of Green's functions $G$ and self-energies $\Sigma$ are implicit):

$$
\mathrm{T} \cdot \mathrm{G}=-\mathrm{i}[1-(\omega-\mathrm{i} \mu) \mathrm{G}-\Sigma \cdot \mathrm{G}], \mathrm{G} \cdot \mathrm{T}=-\mathrm{i}[1-(\omega-\mathrm{i} \mu) \mathrm{G}-\mathrm{G} \cdot \Sigma] \text {, }
$$

$$
\begin{gathered}
\mathrm{T} \cdot \mathrm{G} \cdot \mathrm{T}=-\mathrm{i} \mathrm{T}+\Sigma-\Sigma \cdot \mathrm{G} \cdot \Sigma+(\omega-\mathrm{i} \mu)(1-\Sigma \cdot \mathrm{G}-\mathrm{G} \cdot \Sigma)-(\omega-\mathrm{i} \mu)^{2} \mathrm{G}, \\
{[\mathrm{T} ; \varrho]=\operatorname{Tr}_{\omega}[\Sigma ; \mathrm{G}],(11)}
\end{gathered}
$$


where we have introduced the notation

$$
\frac{1}{\beta} \sum \omega \mathrm{e}^{\mathrm{i} \omega 0+} \mathrm{f}(\mathrm{i} \omega) \cdot \mathrm{g}(\mathrm{i} \omega) \equiv \mathrm{T} \omega(\mathrm{f} \cdot \mathrm{g}) \cdot(12)
$$

Applying Eqs. (12) to Eq. (10), we obtain

$$
\begin{aligned}
& F_{o \alpha, o \prime \alpha \prime}^{X Y}=\frac{1}{2} \delta_{\text {oo, }} \operatorname{Tr}_{\omega} \operatorname{Tr}_{\mathrm{m}, \sigma}\left[\left(S_{o \alpha}^{X} ; S_{o \alpha}^{Y}\right) \cdot(G ; \Sigma)_{\mathrm{o}}^{\mathrm{o}}\right]-\operatorname{Tr}_{\omega} \operatorname{Tr}_{m, \sigma} \\
& {\left[S_{o \alpha}^{X} \cdot[G ; \Sigma]_{0}^{0 \prime} \cdot S_{o \prime \alpha}^{Y} \cdot(G ; \Sigma)_{\mathrm{o}}^{\mathrm{o \prime}}+S_{o \alpha}^{X} \cdot[G ; \Sigma]_{\mathrm{o},}^{\mathrm{o}} \cdot S_{o \prime \alpha,}^{Y} \cdot[\Sigma \cdot G]_{o}^{o \prime}\right.} \\
& \left.-S_{o \alpha}^{X} \cdot[\Sigma \cdot G \cdot \Sigma]_{o^{\prime}}^{o} \cdot S_{o^{\prime} \alpha}^{Y} \cdot \mathrm{G}_{\mathrm{o}}^{\mathrm{o \prime}}-S_{o \alpha}^{X} \cdot \mathrm{G}_{o^{\prime}}^{o} \cdot S_{o^{\prime} \alpha}^{Y} \cdot[\Sigma \cdot G \cdot \Sigma]_{o}^{o \prime}\right]-T r_{\omega} T r_{m, \sigma} \\
& \left(S_{o \alpha}^{X} \cdot \Sigma_{o \prime}^{o} \cdot S Y o^{\prime} a^{\prime} \cdot G_{o}^{o \prime}+S_{o \prime \alpha}^{Y} \cdot \Sigma_{o}^{o \prime} \cdot S_{o \alpha}^{X} \cdot G_{o \prime}^{o}\right),(13)
\end{aligned}
$$

We then consider the magnetic parameters determined from the first-order response. From Eqs.(68) of Ref. [12], we see that these are $B_{0}^{x}, B_{0}^{y}, D_{0}^{x} O^{\prime}, D_{0}^{y} O^{\prime}, C_{0}^{x} O^{\prime}$, and $C_{0}^{y} O^{\prime}$. The first-order response term can be written as

$$
V_{o \alpha}^{X}=i \operatorname{Tr}_{m, \sigma}\left[S_{o \alpha}^{X} \cdot[\rho ; T]_{\mathrm{o}}^{\mathrm{o}}\right]=i \operatorname{Tr}_{m, \sigma} \operatorname{Tr}_{\omega}\left[S_{o \alpha}^{X} \cdot[G ; \Sigma]_{\mathrm{o}}^{\mathrm{o}}\right]=i \operatorname{Tr}_{m, \sigma} \operatorname{Tr}_{\omega} \sum_{o r}\left[S_{o \alpha}^{X} \cdot\left[G_{0 \prime}^{0} \cdot \Sigma_{0}^{0 \prime}-\Sigma_{0 \prime}^{0} \cdot G_{0}^{0 \prime}\right]\right.
$$

By separating local and non-local terms, as well as taking into account the symmetries of the latter, it is then possible to identify the remaining parameters of the spin model. It should be noted that the parameters obtained with this procedure are not equivalent to those expressed in terms of the singleelectron Hamiltonian $T$ in Refs. $[11,12,17]$. These are different definitions, which respect the defining equations (68) of Ref. $[11,12,17]$, but are more directly applicable for a DMFT implementation.

\section{Conclusions}

We have provided the formulas for the general exchange tensor expressing the quadratic magnetic interactions in strongly correlated systems, in a version that can be implemented via DMFT. The formulas allow to compute the effects due to intrinsic-spin and orbital degrees of freedom of the electrons on equal footing (the orbital magnetic moments are not quenched), and to distinguish between spin, orbital and spin orbital interactions that contribute to the exchange tensor. The obtained formulas represent the extension to the relativistic case and the generalization to unquenched orbital magnetic moments of the well-known formulas for spin-only exchange interactions, which are recovered as a particular case. We remark that effects due to the non-locality of self-energies in position space are included in our theory both as presented in Ref. [12] and as presented here; although they cannot be computed within DMFT, a possible approach to include them is via the Dual-Fermion scheme.

\section{Competing Interests}

The author declared that no conflict of interest exists.

\section{How to Cite this Article}

Eunsung Jekal, "External Environment Dependent Spin and Orbital Exchange Interactions", J. Mod. Sim. Mater., vol. 3, no. 1, pp.79-83, Jul. 2020. https://doi.org/10.21467/jmsm.3.1.79-83

\section{References}

[1] B. D. Serot and J. D. Walecka, Adv. Nucl. Phys.16, 1(1984).

[2] R. Jastrow, Physical Review, 98, 1479 (1955).

[3] B. H. Brandow, Reviews of Modern Physics, 39, 771 (1967).

[4] K. Huang and C. N. Yang, J. M. Luttinger, Physical review, 105, 767 (1957).

[5] B. Sutherl, Physical Review A, 4, 2019 (1971).

[6] E. H. Lieb, Physical review letters, 62, 1201 (1989).

[7] A. Georges and G. Kotliar, Physical Review B, 45, 6479 (1992). 
Eunsung Jekal, J. Mod. Sim. Mater.; Vol. 3 Issue 1, pp: 79-83, 2020

[8] K. Held, I. Nekrasov, G. Keller, V. Eyert, N. Blumer, A. Mc Mahan, R. Scalettar, T. Pruschke, V. Anisimov, and D. Vollhardt, physica status solid, 243, 2599 (2006).

[9] H. Zhang, K. Haule, and D. Vanderbilt, Physical review letters, 111, 246402 (2013).

[10] J. Minar, L. Chioncel, A. Perlov, H. Ebert, M. Katsnel-son, and A. Lichtenstein, Physical Review B, 72, 045125 (2005).

[11] A. Alvarez-Arenal, J. A. Alvarez-Riesgo, J. M. P. A-Lopez, and J. P. Fernandez-Vazquez, Community dentistry and oral epidemiology, 26, 166 (1998).

[12] N. Tsuji, T. Oka and H. Aoki, Physical Review B, 78, 235124 (2008).

[13] M. M. Desjardins, et al., Nature Materials 18, 1060 (2019).

[14] S. Tsesses, C. Kobi, O. Evgeny, G. Bergin, and B. Guy, Nano letters 19, 4010 (2019).

[15] W. Lin, L. Lei, D. Fatih, L. Changjian, R. Hélène, Y. Xiaojiang, Z. Bangmin et al., Nature communications 10, 1 (2019).

[16] Q. Chen, L. Lei, P. Georgia, W. Puhua, L. Jianming, G. Theodosis, T. Eleni, G. Dimitrios, R. Petra, and Y. Jianting, Nano letters 19, 1520 (2019).

[17] K. F. Mak, K. L. McGill, J. Park, P. L. McEuen, Science, 344, 1489 (2014).

[18] Z. Wu, B. T. Zhou, X. Cai, P. Cheung, G. B. Liu, M. Huang, J. Lin, T. Han, L. An, Y. Wang, et al., Nat. Commun. 10, 611 (2019).

[19] S. Xu, J. Shen, G. Long, Z. Wu, Z. Q. Bao, C. C. Liu, X. Xiao, T. Han, J. Lin, Y. Wu, et al., Phys. Rev. Lett. 118, 067702 (2017).

[20] H. C. P. Movva, B. Fallahazad, K. Kim, S. Larentis, T. Taniguchi, K. Watanabe, S. K. Banerjee, E. Tutuc, Phys. Rev. Lett. 118, 247701 (2017).

[21] T. S. Ghiasi, J. Ingla-Ayne, A. A. Kaverzin, B. J. van Wees, Nano Lett. 17, 7528 (2017).

[22] J. O. Island, X. Cui, C. Lewandowski, J. Y. Khoo, E. M. Spanton, H. Zhou, D. Rhodes, J. C. Hone, T. Taniguchi, K. Watanabe, et al., Nature, 571, 85 (2019).

[23] J. Nitta, T. Akazaki, H. Takayanagi, T. Enoki, Phys. Rev. Lett. 78, 1335 (1997).

[24] T. Koga, J. Nitta, T. Akazaki, H. Takayanagi, Phys. Rev. Lett. 89, 046801 (2002).

[25] G. Chang, B. J. Wieder, F. Schindler, D. S. Sanchez, I. Belopolski, S. M. Huang, B. Singh, D. Wu, T. R. Chang, T. Neupert, et al.,Nat. Mater. 17, 978 (2018).

Publish your research article in AIJR journals-

* Online Submission and Tracking

* Peer-Reviewed

* Rapid decision

* Immediate Publication after acceptance

* Articles freely available online

* Retain full copyright of your article.

Submit your article at journals.aijr.in
Publish your books with AIJR publisher-

* Publish with ISBN and DOI.

* Publish Thesis/Dissertation as a Book.

* Publish Monograph.

* Publish Edited Volume/ Book.

* Publish Conference Proceedings

- Retain full copyright of your books.

Submit your manuscript at books.aijr.org 